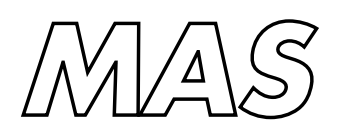

Modelling, Analysis and Simulation

Modelling, Analysis and Simulation
MAS An improved front tracking method for the Euler
equations

J.A.S. Witteveen, P.G. Bakker, B. Koren

RePORt MAS-E0605 JanUARY 2006 
Centrum voor Wiskunde en Informatica (CWI) is the national research institute for Mathematics and Computer Science. It is sponsored by the Netherlands Organisation for Scientific Research (NWO).

CWI is a founding member of ERCIM, the European Research Consortium for Informatics and Mathematics.

CWI's research has a theme-oriented structure and is grouped into four clusters. Listed below are the names of the clusters and in parentheses their acronyms.

Probability, Networks and Algorithms (PNA)

Software Engineering (SEN)

\section{Modelling, Analysis and Simulation (MAS)}

Information Systems (INS)

Copyright (C) 2006, Stichting Centrum voor Wiskunde en Informatica

P.O. Box 94079, 1090 GB Amsterdam (NL)

Kruislaan 413, 1098 SJ Amsterdam (NL)

Telephone +31205929333

Telefax +31205924199

ISSN 1386-3703 


\title{
An improved front tracking method for the Euler equations
}

\author{
ABSTRACT \\ In this paper an improved front tracking method for the Euler equations is presented. The \\ method is improved by assigning wave types to the fronts. These wave types determine the \\ velocities of the fronts and their mutual interactions. This results in a physically more consistent, \\ a more accurate and a faster simulation. The method is applied to the Euler equations for one- \\ dimensional unsteady and supersonic two-dimensional steady flow.
}

2000 Mathematics Subject Classification: 35L67, 65M25, 76J20, 76L05

Keywords and Phrases: front tracking; gas dynamics; Euler equations; supersonic flows; shock waves; rarefaction waves; contact discontinuities 



\title{
An Improved Front Tracking Method for the Euler Equations
}

\author{
J.A.S. Witteveen, P.G. Bakker ${ }^{\dagger}$ and B. Koren ${ }^{\dagger}$ \\ Delft University of Technology, The Netherlands
}

\begin{abstract}
In this paper an improved front tracking method for the Euler equations is presented. The method is improved by assigning wave types to the fronts. These wave types determine the velocities of the fronts and their mutual interactions. This results in a physically more consistent, a more accurate and a faster simulation. The method is applied to the Euler equations for one-dimensional unsteady and supersonic two-dimensional steady flow.
\end{abstract}

\section{Introduction}

Many problems in physics are governed by conservation laws. The mathematical formulation of these conservation laws often results in hyperbolic partial differential equations of which the solutions can contain discontinuities. For fixed-grid methods these discontinuities are hard to resolve accurately. As opposed to that, front tracking methods can accurately resolve discontinuities quite easily. These methods resolve discontinuities in the solution as true discontinuities by treating them as extra degrees of freedom. A diversity of research has been devoted to front tracking methods. Theoretical work, including proofs of stability and convergence, has been done by Bressan. ${ }^{1}$ The numerical implementation of the front tracking method is also well studied. Its application to hyperbolic conservation laws in one dimension has been presented by Langseth, Risebro and Tveito. ${ }^{2,3,4,5}$ The method has been extended to more spatial dimensions by dimensional splitting in combination with grid adaption by Lie $^{6}$ and Holden. ${ }^{7}$

Nowadays, two mainstreams in front tracking methods can be identified. First, methods which use front tracking for discontinuities combined with a fixed-grid method for resolving the continuous phenomena, see Glimm, McBryan and co-workers. ${ }^{8}$ Secondly, methods which do not use such an additional fixed-grid method. The latter methods discretize continuous phenomena by small discontinuities resulting in a piecewise constant step-function approximation like in Ref. 2-5. The improved front tracking method presented here is of the latter class of front tracking methods.

The general concept of front tracking methods is described in the context of a general hyperbolic conservation law for scalars

$$
\frac{\partial u}{\partial t}+\frac{\partial f(u)}{\partial x}=0, \quad u(x, 0)=u_{0}(x) .
$$

The most fundamental problem for this equation is the Riemann problem which is defined by the following initial condition:

$$
u_{0}(x)= \begin{cases}u_{1}, & x<0 \\ u_{\mathrm{r}}, & x>0\end{cases}
$$

This problem can have a classical continuous solution or a weak discontinuous solution. A standard front tracking method solves the Riemann problem at $t=0$ exactly and determines the fronts that are created at

${ }^{*}$ Ph.D. student, Department of Aerospace Engineering, P.O. Box 5058, 2600 GB Delft, The Netherlands, Student Member AIAA.

${ }^{\dagger}$ Professor, Department of Aerospace Engineering, P.O. Box 5058, 2600 GB Delft, The Netherlands, Senior Member AIAA.

$$
1 \text { of } 10
$$

American Institute of Aeronautics and Astronautics 
the initial discontinuity. This solution can be either a shock wave or a rarefaction wave. If the solution is a shock wave it is discretized exactly by one front with a velocity equal to the shock velocity. If the solution is a continuous rarefaction wave the continuous phenomenon is approximated by a series of step functions. Multiple fronts are introduced at which the solution changes discontinuously resulting in a piecewise constant approximation of the rarefaction wave, which is of first order in the $L^{1}$ norm. ${ }^{3}$

A more general initial value problem is the Cauchy problem for which the initial condition is defined as

$$
u(x, 0)=u_{0}(x) \text {. }
$$

First, assume that $u_{0}(x)$ is piecewise constant. Then a standard front tracking method solves this problem by solving local Riemann problems at the discontinuities in the initial condition. At these positions fronts are created describing the solution of the local Riemann problems. If such a solution consists of a discontinuity, only one front is created and if the solution is continuous a fan of fronts is created to approximate the continuous solution with a series of step functions. The difference between a Cauchy problem and the Riemann problem is that for nonlinear equations the fronts created by the initial condition can intersect or collide with each other at a later time. At such an intersection of two or more fronts, these fronts interact. Because at the intersection point the solution resembles a local Riemann problem, the interaction of fronts is determined by solving that local Riemann problem. Due to this interaction new fronts are created and they can collide with other fronts at a later time and so on.

Secondly, a continuous initial condition is considered. This initial condition has to be approximated by a series of piecewise constant step functions, which results in a first order error. Further the solution procedure is the same as described above.

Before presenting the improved front tracking method, we consider the application of a standard front tracking method to the Euler equations for one-dimensional unsteady flows like in Ref. 3. These equations are given by

$$
\begin{aligned}
& \frac{\partial U}{\partial t}+\frac{\partial F(U)}{\partial x}=0, \quad U(x, 0)=U_{0}(x), \\
& U=\left(\begin{array}{c}
\rho \\
\rho u \\
\rho E
\end{array}\right), \quad F=\left(\begin{array}{c}
\rho u \\
p+\rho u^{2} \\
\rho u H
\end{array}\right),
\end{aligned}
$$

with $\rho$ the density, $u$ the velocity, $p$ the pressure, $E$ the internal energy, which for a perfect gas reads $E=\frac{1}{\gamma-1} \frac{p}{\rho}+\frac{1}{2} u^{2}$, with $\gamma$ the ratio of specific heats $\left(\gamma=\frac{c_{\mathrm{p}}}{c_{\mathrm{v}}}\right)$, and with $H$ the enthalpy, $H=E+\frac{p}{\rho}$. The principles for solving this problem are the same as in the scalar case. The Riemann problem again plays a key role in the front tracking algorithm. The solution of a Riemann problem for the Euler equations generally consists of three waves: A left and right running shock or rarefaction wave and a contact discontinuity in between. So, four different constant states can be identified in the solution of this problem, see figure 1, where the two post-states only differ in their density $\rho_{\mathrm{ml}}$ and $\rho_{\mathrm{mr}}$. The pressure and the velocity in the post-states $p_{\mathrm{m}}$ and $u_{\mathrm{m}}$ can be solved for in the $p, u$-state space by determining the intersection point of the two curves representing the left and the right running waves. A non-isentropic compression by means of a shock wave is represented by the Hugoniot curve

$$
\Delta p= \pm m \Delta u, \quad m=\rho_{\text {pre }} c_{\text {pre }} \sqrt{1+\frac{\gamma+1}{2 \gamma} \frac{p_{\text {post }}-p_{\text {pre }}}{p_{\text {pre }}}}
$$

where $m$ is the mass flux through the shock wave, $c$ the speed of sound and where the subscripts pre and post refer to the flow upstream and downstream of the shock, respectively. A rarefaction wave is represented by the Poisson curve

$$
\Delta p= \pm m \Delta u, \quad m=\rho_{\mathrm{pre}} c_{\mathrm{pre}} \frac{\gamma-1}{2 \gamma} \frac{1-\frac{p_{\mathrm{post}}}{p_{\mathrm{pre}}}}{1-\left(\frac{p_{\mathrm{post}}}{p_{\mathrm{pre}}}\right)^{\frac{\gamma-1}{2 \gamma}}}
$$




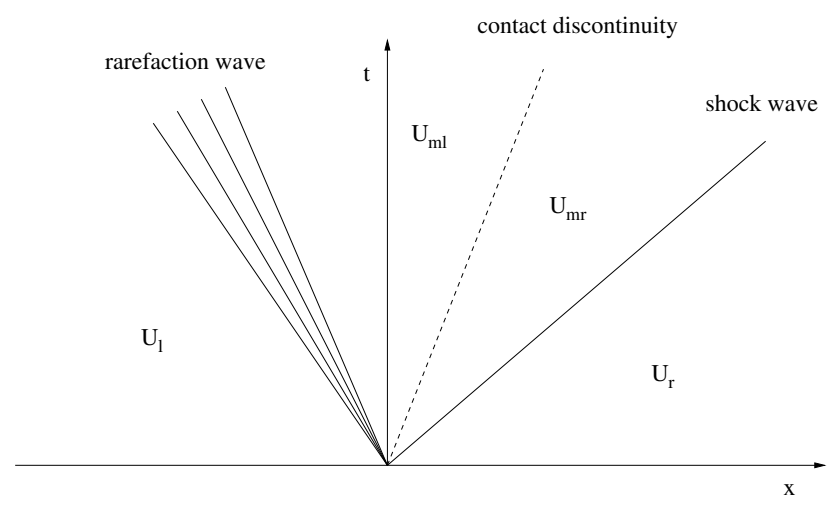

Figure 1. A typical solution of the Riemann problem for the Euler equations for one-dimensional unsteady flow

which describes an isentropic change of states. Note that the Poisson curve can also be used for describing an isentropic compression. In general, the Riemann problem has to be solved iteratively, after which the solution in terms of wave fronts can be constructed. Again one front is used for a discontinuous shock wave or contact discontinuity and a rarefaction wave is discretized by multiple fronts. See Ref. 3 for an example how to discretize a rarefaction wave.

Next, consider the application of the standard front tracking method to the Cauchy problem for the Euler equations in one dimension. First, the initial condition is approximated by a piecewise constant step function. Secondly, local Riemann problems are solved at the discontinuities in the approximate initial condition resulting in shock waves, contact discontinuities and rarefaction waves, which are discretized by wave fronts. These fronts can intersect for $t>0$. At these intersection points local Riemann problems are solved to determine the wave interactions resulting in new fronts describing shock waves, contact discontinuities and rarefaction waves in the solution of the local Riemann problem just like for the initial solution and so on.

\section{The improved front tracking method}

A front tracking method is in general a very physical way to simulate the flow. However, the current formulation of the method allows some non-physical behavior, for example: (i) the method is unable to resolve isentropic compressions - which can occur in real flow fields - because a compression in the local Riemann problems is always represented by a non-isentropic shock wave, (ii) after every wave interaction a rarefaction wave is represented by a centered expansion fan like in a standard Riemann problem. However, in general this is physically inaccurate, because whether a rarefaction wave is created in the form of a centered expansion fan depends on the type of waves that interact. The improvements which are presented below concern especially these two points.

The front tracking method is extended by introducing wave types which are assigned to the fronts. First, these wave types are used to determine the correct velocities of the fronts. Secondly, the solution of the local Riemann problems does no longer depend on the flow conditions to the left and the right of the intersection point only, but also on the wave types of the intersecting fronts. Therefore, several different Riemann solvers are introduced. In Ref. 4 already four different Riemann solvers were introduced for decreasing the computational work of the method which is highly dominated by solving the Riemann problems. The Riemann solvers introduced here differ from those introduced in the latter reference. 


\section{A. The wave types}

Discontinuities in the solution of the Riemann problem like shock waves and contact discontinuities are discretized exactly by a single front. These two waves are assigned the according wave type of shock wave (sw) and contact discontinuity (cd). First, these wave types determine the velocity of the corresponding wave. The shock wave velocity $u_{\mathrm{s}}$ is given by the Rankine-Hugoniot relation

$$
u_{\mathrm{s}}\left[U_{\text {post }}-U_{\text {pre }}\right]=\left[F_{\text {post }}-F_{\text {pre }}\right],
$$

and the velocity of the contact discontinuity is given by the velocity $u_{\mathrm{m}}$ from the Riemann solution, see figure 1. Secondly, the wave types of the fronts influence their interaction with other waves.

The fronts which can discretize a rarefaction wave are assigned three different wave types which can be associated with characteristics of the rarefaction wave: the leftmost characteristic (lc), the rightmost characteristic (rc) and an interior characteristic (ic). Again the fronts with different wave types are assigned different relations for their velocities:

$$
\begin{aligned}
& u_{\mathrm{lc}}=(u \pm c)_{\mathrm{l}}, \\
& u_{\mathrm{rc}}=(u \pm c)_{\mathrm{r}}, \\
& u_{\mathrm{ic}}=\frac{1}{2}\left((u \pm c)_{1}+(u \pm c)_{\mathrm{r}}\right),
\end{aligned}
$$

where the subscripts 1 and $\mathrm{r}$ denote the flow conditions immediately at the left and the right of the front, respectively. Each of these three wave types also interacts differently with other fronts. By assigning these front velocities the exact dimension of the expansion fans is obtained. The interior wave fronts of the expansion fan can be distributed in various ways. In Ref. 3 an equidistant parameterization of the Poisson curve is used. Here it is chosen to vary the velocity of the fronts with uniform steps inside the rarefaction wave resulting in a minimization of the velocity approximation error.

Finally, in a standard Riemann problem only discontinuous variations of the entropy over a contact discontinuity can occur. However, in the solution of a Cauchy problem continuous variations of the entropy can exist. For example, consider a left running shock wave intersecting a right running continuous rarefaction wave, then the shock strength in terms of entropy loss changes continuously during the interaction resulting in a continuous change of entropy behind the shock wave, see figure 2.

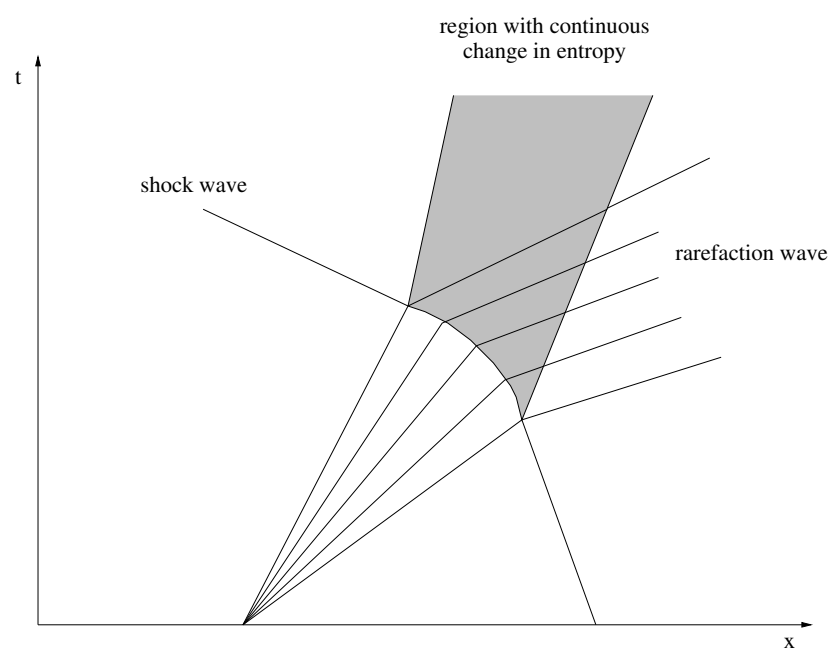

Figure 2. The creation of a region with a continuous change in entropy by the interaction of a left running shock wave and a right running rarefaction wave

4 of 10

American Institute of Aeronautics and Astronautics 
To discretize this phenomenon accurately three more wave types are needed. In a standard front tracking method this continuous change of entropy is discretized by a series of fronts resulting in a piecewise constant approximation of the variation in entropy. Here the continuous change in entropy is discretized in the same way, however, the fronts are not assigned the contact discontinuity wave type. These fronts represent a continuous phenomenon and not a contact discontinuity which affects their interaction with other fronts.

Consider, for example, a left running shock wave which intersects a contact discontinuity, see figure 3 a. In that case a shock wave continues at the left of the contact discontinuity and a shock wave or a centered expansion fan is reflected at the right of the contact discontinuity. However, if the left front is actually the right end of a continuous change of entropy (figure $3 \mathrm{~b}$ ), the shock wave will reflect as the right end of an isentropic expansion or compression fan with wave type rc. Therefore, the fronts discretizing such a continuous change of entropy have to be assigned other wave types than the true contact discontinuity (cd), but - instead - similar to the fronts representing a rarefaction wave: the leftmost front (lcd), the rightmost front ( $\mathrm{rcd}$ ) and an interior front (icd) of a continuous change of entropy. These wave types are all assigned the same velocity $u_{\mathrm{m}}$, which is the velocity in the middle state of the solution of the local Riemann problem.

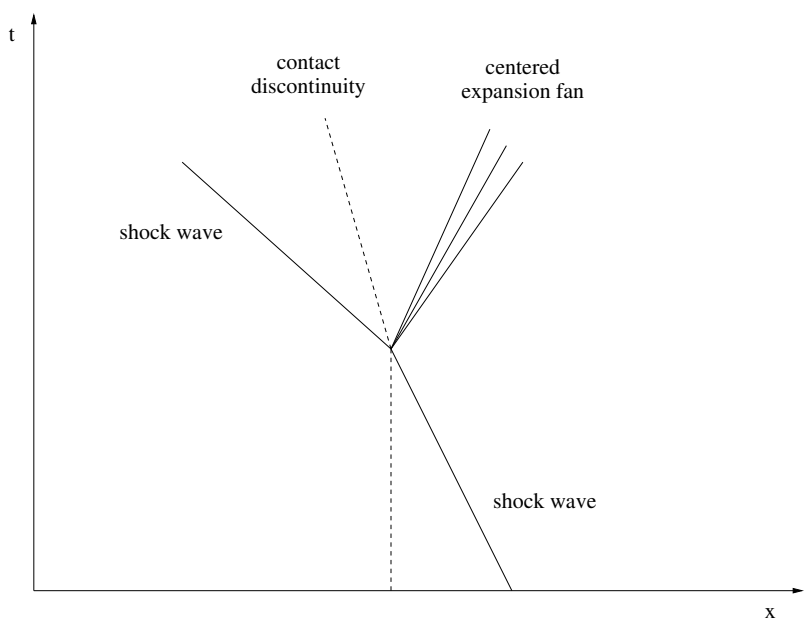

(a) interaction with a contact discontinuity

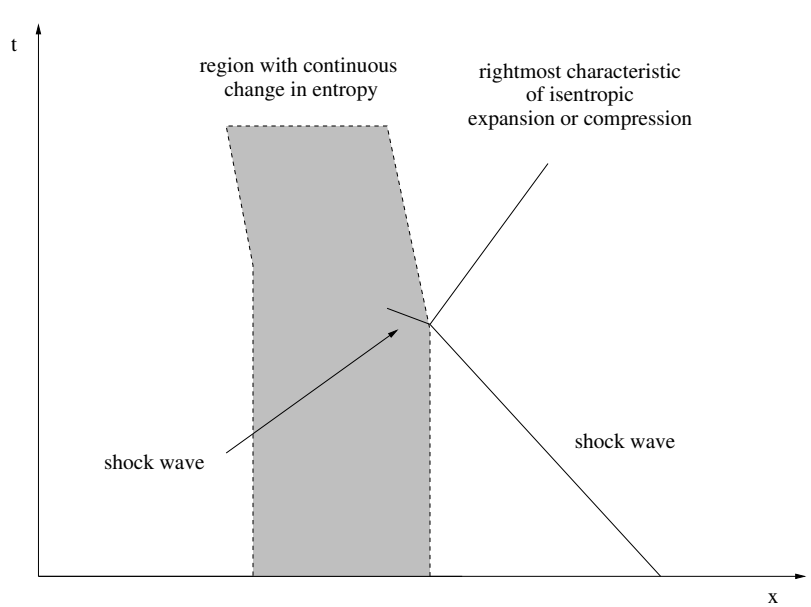

(b) interaction with a continuous change of entropy

Figure 3. The interaction of a left running shock wave and (a) a contact discontinuity and (b) a continuous change of entropy resulting in different reflections

A second important property of the wave fronts is their velocity relative to the flow velocity. Shock waves and rarefaction waves can run to the left or to the right with respect to the fluid depending on whether they are created as left or right waves in a local Riemann problem. All contact discontinuities have the same velocity as the fluid. So, all fronts can also be assigned one of the following three families of waves: left running waves $(-1)$, right running waves $(1)$ and waves which have no relative velocity with respect to the surrounding fluid (0).

Summarizing, the total number of waves front types is 8: 1 shock wave type (sw), 3 wave types representing characteristics (lc, rc, ic) and 4 wave types representing change of entropy (cd, lcd, rcd, icd). Furthermore, there are 3 wave front families with respect to their velocity relative to the fluid. Combining these two subdivisions of fronts, in total 12 different wave front types in combination with relative velocity families can be identified: 2 shock wave types, 6 characteristics and 4 wave types representing change of entropy. 
Notice that these wave types can also be used to discretize continuous initial and boundary conditions more accurately by taking into account that discontinuities in the piecewise constant approximation of the initial and boundary conditions can resemble continuous phenomena.

\section{B. The wave interactions}

The wave types are not introduced to accurately simulate the wave velocities only, but also to simulate the wave interactions between the fronts correctly. In a standard front tracking method the interaction of two or more waves is determined by solving a local Riemann problem in the intersection point. Its solution is interpreted as in a standard Riemann problem such that a compression is always a shock wave, an expansion is always a centered expansion fan and the middle wave is always a contact discontinuity. However, this is physically not the most accurate representation in case of interactions between wave fronts. Taking the wave front types and the relative velocity families of the intersecting waves into account results in a more accurate simulation of the wave interactions.

Using gas dynamics theory the interactions between all different wave types introduced above can be solved for by considering the wave types of the intersecting fronts. Actually, the wave types of the fronts created at an interaction can, in general, be determined before solving the local Riemann problem itself. For example, if two left running characteristics intersect, a left running shock wave, a rightmost right running characteristic (rc) and a rightmost continuous change in entropy (rcd) will be created. Remind that characteristics do not occur at their own in the flow field, they are always part of a compression or expansion fan of characteristics.

The first step in determining the resulting wave patterns for all possible wave interactions is to determine which wave intersections can actually occur in a flow field. In principle, the maximum of possible wave intersections is the square of the number of waves $12^{2}=144$. However, not all of these wave interactions can occur in a real flow field. It follows that only 70 can. For example, fronts with relative velocity family (0) can never intersect each other.

The second step is to determine the resulting wave patterns of all possible wave interactions. This includes prescribing the left, right and middle wave type of the solution of the local Riemann problem choosing from the 12 available wave types in combination with the relative velocity family. Most of these wave types can be prescribed before solving the Riemann problem. The only exception is the shock wave, because this wave changes into a centered expansion fan if the solution of the Riemann problem prescribes a rarefaction wave.

This results in a wave dynamics matrix describing the physical behavior of the Euler equations in terms of its wave dynamics. With this wave dynamics matrix it can be determined whether the waves created at an interaction are isentropic or possibly non-isentropic before solving the local Riemann problem. This knowledge has to be taken into account in solving the local Riemann problems.

\section{The Riemann solvers}

In a standard Riemann problem, the wave types present in the solution are not known before solving it. Therefore, whether isentropic or non-isentropic waves are present in the solution is determined during the iterative solution process. The Riemann problem can be solved in the $p, u$-state space. The solution is the intersection point of the curves representing the left and the right wave. Non-isentropic shock waves are represented by the Hugoniot curve (4) and isentropic waves are represented by the Poisson curve (5) in the $p, u$-state space. In general, solving the Riemann problem requires an iteration process.

In our case, in general, the wave types of the fronts created at the local Riemann problem are known before solving it. Therefore, the representation of the waves in the $p, u$-state space can be chosen a priori. This implies that four different Riemann solvers have to be implemented and one of these has to be selected, either: (i) the normal Riemann solver in which a wave is represented by a Hugoniot curve if it represents a compression and by a Poisson curve if it represents an expansion, or (ii) the isentropic Riemann solver in which both waves are represented by Poisson curves and which has an analytical solution, or (iii) a Riemann

$$
6 \text { of } 10
$$

American Institute of Aeronautics and Astronautics 
solver in which the left wave is isentropic and represented by a Poisson curve and the right wave is represented by either a Hugoniot or a Poisson curve or (iv) like (iii) but the other way around.

The computational time of the front tracking method is dominated by solving the Riemann problems. The introduction of the wave types reduces the number of non-isentropic compression waves by replacing them when appropriate - in a physically consistent way by isentropic compression waves. Therefore, the isentropic Riemann solver (ii), which has an analytical solution, can be used more often. So, the average number of iterations per Riemann problem is reduced compared to a standard front tracking method. For Riemann solvers (i), (iii) and (iv) an efficient iterative algorithm is used similar to that described by Emanuel. ${ }^{9}$

\section{The numerical results}

We present numerical results for three test problems. The first one is a flow problem with one-dimensional unsteady Euler flow. The other two are supersonic two-dimensional steady Euler flows around two different wing sections.

\section{A. The blast wave test problem}

The blast wave test problem of Woodward and Collela ${ }^{10}$ is considered. This one-dimensional unsteady initial-boundary value problem is ideally suited to investigate the performance of a numerical method with respect to the resolution of strong shock waves, expansion fans and contact discontinuities and their mutual interactions.

The one-dimensional spatial domain has unit length and the fluid is enclosed by a reflecting wall at both ends. Initially, the fluid is divided into three uniform domains with zero velocity, unit density and pressure

$$
p= \begin{cases}1000 & 0<x<0.1 \\ .01 & 0.1<x<0.9 \\ 100 & 0.9<x<1\end{cases}
$$

From these discontinuities in the initial condition, two shock waves and two contact discontinuities run towards the center of the domain and two centered expansion fans run towards the walls at which they reflect, such that all these flow phenomena interact with each other in the interior of the flow domain. The initial and boundary conditions of this test problem have been chosen in such a way that they illustrate the strong dependence of the accuracy of the overall flow solution on accurately resolving the discontinuities in the flow field.

The accuracy of the front tracking method can be influenced by prescribing the value of several constants. First, the iteration for solving the Riemann problem is stopped if the difference between the two pressures is smaller than $10^{-6}$. Secondly, a wave is neglected if the change of flow conditions over a wave is smaller than one percent to avoid resolving many insignificant waves. Finally, the number of waves which represent a centered expansion fan is taken constant. This number has a major influence on the accuracy of the solution.

In figure 4, the solution of the test problem in terms of the waves in the space-time domain, which is equal to the grid, is shown for a discretization of the centered expansion fans with 10 waves. In figure 5 the velocity and the density distribution at $t=0.038$ is shown for 30 waves per centered expansion fan. By comparing these two figures with results in Ref. 10, it can be concluded that the solution including the position of the waves is very accurate. In these figures we have used an a posteriori linearization step in the appropriate cells to represent the solution piecewise linearly.

Notice that the solution has been obtained by using a very small number of cells to discretize the flow domain in space-time, namely for 30 waves per centered expansion fan the total number of space-time cells is only 4374. The computational time is dominated by solving the Riemann problems in the intersection points. In total 4147 Riemann problems have to be solved. The average number of iterations per Riemann problem is only 1.19 , because many wave interactions are described by two Poisson curves so that the solution can be determined analytically. 


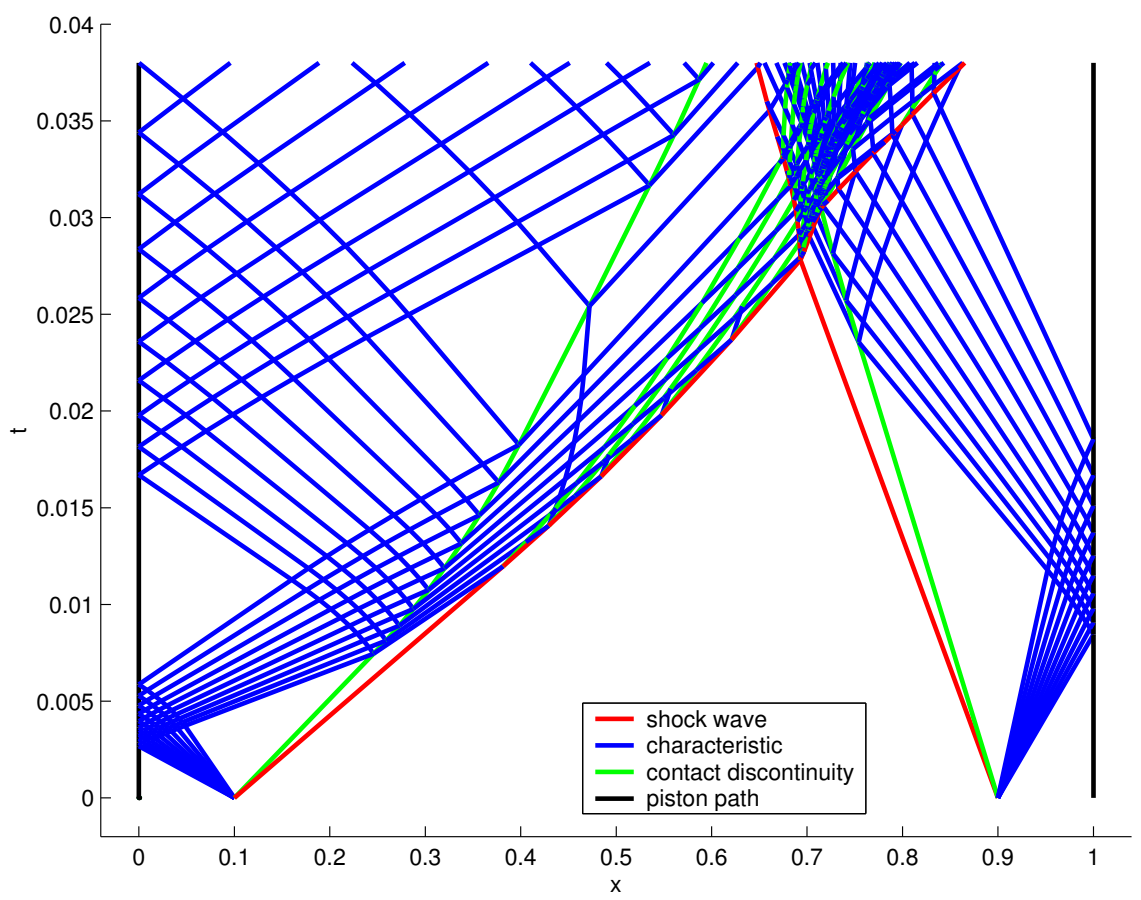

Figure 4. The solution for the two interacting blast waves test problem in space-time obtained by the front tracking method with 10 waves per centered expansion fan

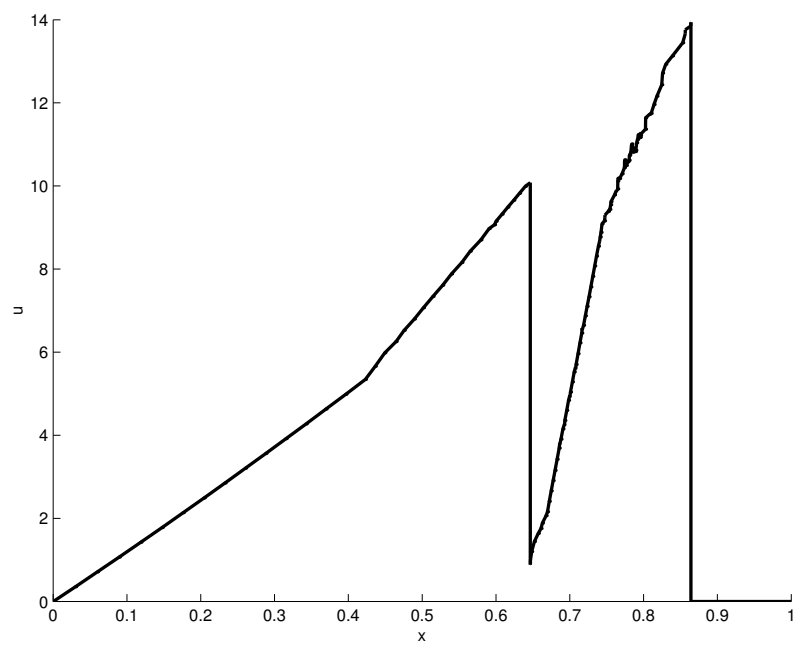

(a) velocity

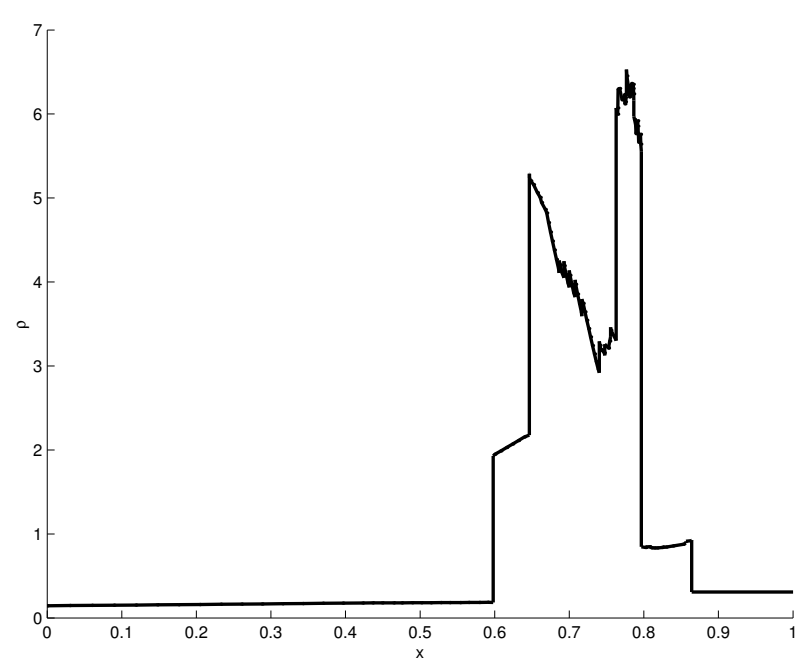

(b) density

Figure 5. The solution for (a) the velocity and (b) the density of the two interacting blast waves test problem at $t=0.038$ obtained by the front tracking method with 30 waves per centered expansion fan 


\section{B. The supersonic two-dimensional steady flow over two wing sections}

In the next test problem, the improved front tracking method is applied to supersonic two-dimensional steady Euler flow around a wing profile. The Euler equations of two-dimensional steady flow are given by

$$
\begin{gathered}
\frac{\partial F}{\partial x}+\frac{\partial G}{\partial y}=0 \\
F=\left(\begin{array}{c}
\rho u \\
p+\rho u^{2} \\
\rho u v \\
\rho u H
\end{array}\right), \quad G=\left(\begin{array}{c}
\rho v \\
\rho u v \\
p+\rho v^{2} \\
\rho v H
\end{array}\right),
\end{gathered}
$$

with $u$ and $v$ the velocity components in $x$ - and $y$-direction, respectively. If the flow is supersonic in the whole flow field, these equations are hyperbolic in the flow direction, such that a front tracking method can be used. For that purpose only minor changes in the algorithm have to be made, because the wave types and the wave interaction dynamics remain the same. The Riemann solvers have to be replaced by solvers for the Riemann problem for supersonic two-dimensional steady flow and an extra variable has to be introduced to describe the second component of the velocity vector.

The results of the front tracking method are compared to a Godunov-type method and an analytical approximation for the shape of the shock waves. The computations with the Godunov-type method are performed with second order accuracy by using a limiter scheme on a $48 \times 48$-grid. Furthermore, a multigrid method is used to speed up the solution process. The code has been written by Koren. ${ }^{11,12}$

In the analytical method, the shock waves are approximated by an isentropic simple wave, such that approximate analytical relations for the shape of the leading and trailing edge shock wave can be derived.

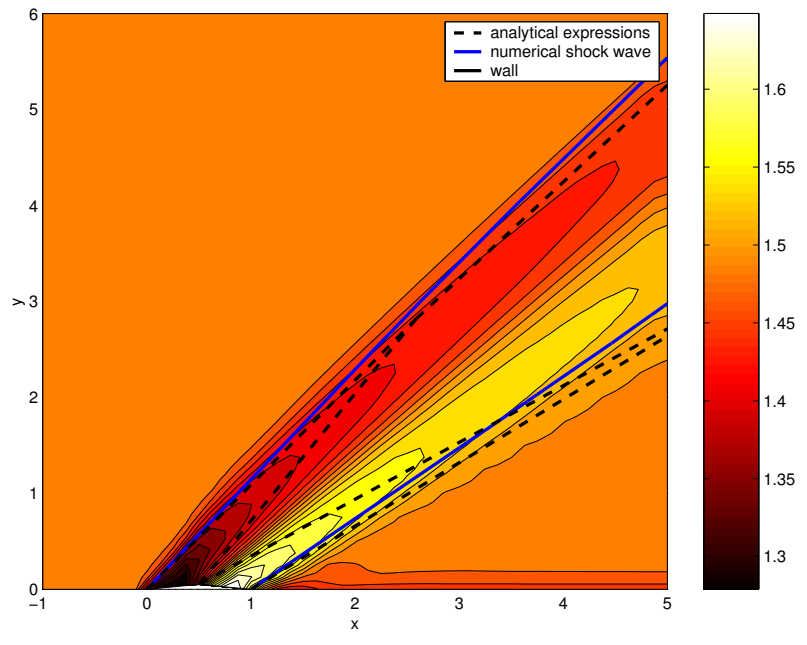

(a) double wedge wing section

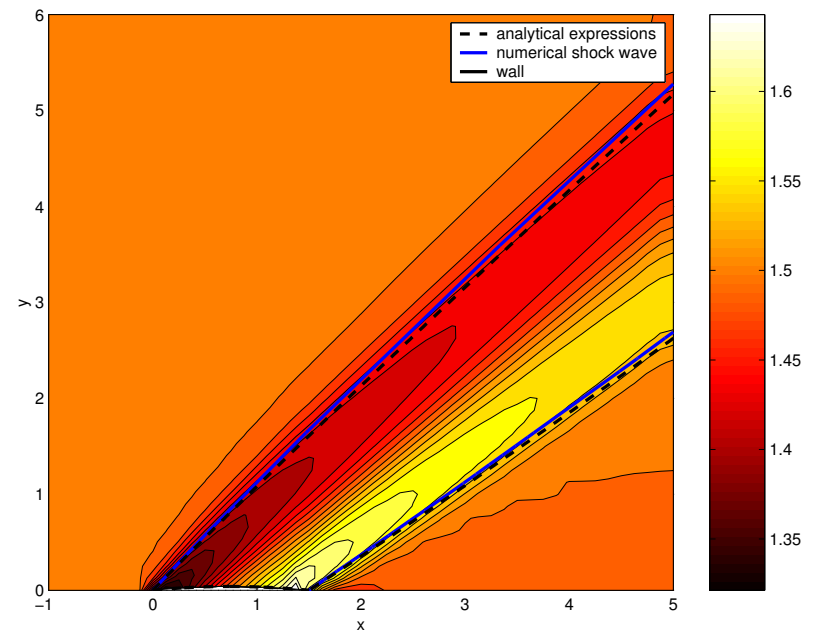

(b) continuously curved wing section

Figure 6. The results for the Godunov-type method in terms of the local Mach number compared to the position of the shock waves according to the front tracking method (-) and the analytical approximation (- -) for (a) the double wedge wing section and (b) the continuously curved wing section, for a free stream Mach number of 1.5 
The two test problems with supersonic two-dimensional steady flow are flows around wing sections. The first wing section is a double wedge wing section and the second one is a continuously curved wing section. The continuously curved wing section is approximated piecewise linearly. However, the discontinuities in the derivative of this piecewise linear function are treated as continuous changes in direction angle (except for the leading and trailing edge). The Mach number of the undisturbed flow is 1.5.

In figure 6, the numerical results of the Godunov-type method in terms of the local Mach number are compared to the position of the shock waves according to the front tracking method and the analytical approximation for the two wing sections. The flow pattern is significantly smeared out by the numerical diffusion in the Godunov-type method, whereas numerical diffusion is absent in the front tracking method.

\section{Conclusions}

We have introduced an improved front tracking method for the Euler equations. The method is faster and more accurate than a standard front tracking method. Wave types are introduced which are assigned to the fronts for a physically more correct simulation. These wave types are used for determining the velocities of the fronts and their mutual interactions. Furthermore, the use of wave types results in a decrease of the average number of iterations per Riemann problem. We have applied the improved front tracking method to test problems with one-dimensional unsteady and supersonic two-dimensional steady Euler flow. The results are excellent with respect to both accuracy and computational efficiency.

\section{References}

${ }^{1}$ A. Bressan, P. LeFloch, Uniqueness of weak solution to systems of conservation laws, Arch. Rational Mech. Anal., 140, 301 (1997).

${ }^{2}$ N.H. Risebro, A. Tveito, Front tracking applied to a nonstrictly hyperbolic system of conservation laws, SIAM J. Sci. Statist. Comput., 12, 1401 (1991). (1992).

${ }^{3}$ N.H. Risebro, A. Tveito, A front tracking method for conservation laws in one dimension, J. Comput. Phys., 101, 130

${ }^{4}$ J.O. Langseth, N.H. Risebro, A. Tveito, A conservative front tracking scheme for 1D hyperbolic conservation laws, in Nonlinear Hyperbolic Problems: Theoretical, Applied, and Computational Aspects (A. Donato et al., Eds.), Notes Numer. Fluid Mech., 43, 385 (Vieweg, Braunschweig, 1993).

${ }^{5}$ J.O. Langseth, On an implementation of a front tracking method for hyperbolic conservation laws, Adv. Engrg. Software, 26, 45 (1996).

${ }^{6}$ K.-A. Lie, V. Haugse, K. Hvistendahl Karlsen, Dimensional splitting with front tracking and adaptive grid refinement, Numer. Methods Partial Differential Eq., 14, 627 (1998).

${ }^{7}$ H. Holden, K.-A. Lie, N.H. Risebro, An unconditionally stable method for the Euler equations, J. Comput. Phys., 150, 76 (1999).

${ }^{8}$ J. Glimm, J. Grove, B Lindquist, O. McBryan, G. Trygvason, The bifurcation of tracked scalar waves, SIAM J. Sci. Stat. Comput., 9, 61 (1988).

${ }^{9}$ G. Emanuel, Analytical Fluid Dynamics (CRC Press, Boca Raton, 2001).

${ }^{10}$ P.R. Woodward, P. Colella, The numerical simulation of two-dimensional flow with strong shocks, J. Comput. Phys., 54, 115 (1984).

${ }^{11}$ B. Koren, Defect correction and multigrid for an efficient and accurate computation of airfoil flows, J. Comput. Phys., 77, 183 (1988).

${ }^{12}$ B. Koren, Euler flow solutions for transonic shock wave-boundary layer interaction, Int. J. Numer. Meth. in Fluids, 9, 59 (1989).

10 of 10 